\title{
Die Jagd nach dem Jungbrunnen
}

\section{In Quest of the Fountain of Youth}

\author{
Autor \\ R. M. Trüeb \\ Institut \\ Dermatologische Klinik, Universitätsspital Zürich
}

\section{Bibliografie}

DOI $10.1055 / \mathrm{s}-2007-966776$

Akt Dermatol 2007; 33:

297-309 @ Georg Thieme

Verlag KG Stuttgart · New York ISSN 0340-2541

Korrespondenzadresse

Prof. Dr. med. Ralph M. Trüeb Dermatologische Klinik Universitätsspital Zürich Gloriastr. 31

8091 Zürich, Schweiz ralph.trueeb@usz.ch

\section{Zusammenfassung}

Immer und überall hat das Alter als letzter Lebensabschnitt und seine Gebrechen die Menschheit beschäftigt und beunruhigt. Philosophen, Naturwissenschaftler und Ärzte haben sich immer darum bemüht, Zusammenhänge aufzudecken, den Alterserscheinungen wirksam vorzubeugen, wenn auch mit begrenztem Erfolg. Es finden sich aus allen Zivilisationen Zeugnisse für zwei Hauptrichtungen: Die erste verfolgt prophylaktische Ziele, um die Jugendfrische zu erhalten und Altersschwäche zu vermeiden; die zweite hat Heilabsichten und fasst sogar die Verjüngung des alternden Menschen ins Auge. Die Hartnäckigkeit, mit der auf der Suche nach dem Jungbrunnen mitunter illusorischen Vorstellungen nachgejagt wurde, erscheint uns bisweilen absurd. Sie beweist aber nur, dass sich der Mensch nicht ohne Weiteres von bestimmten Fantasien zu lösen vermag. Bereits in der Vergangenheit und in allen großen Kulturen wurden aber bemerkenswerte Hypothesen aufgestellt, die seither ihre Bestätigungen fanden. Es sind beispielsweise die Hygiene, Ernährung, Körperkultur, Schönheitspflege, Kosmetik, Eigenverantwortung für die Gesundheit, Vorsorgeuntersuchung, Psychohygiene und Zeitökonomie zu nennen, die heute alle wichtige Elemente des modernen Anti-Aging darstellen. Anti-Aging verfolgt heute als medizinische Fachrichtung systematisch das Ziel der Vorbeugung, Früherkennung, Umkehr und Behandlung altersabhängiger Funktionseinbußen und alterstypischer Veränderungen der Organe und des Gesamtorganismus. Der Zustand der Haut spielt in der Anti-Aging-Medizin eine wichtige Rolle, insofern als Schönheit mit Jugendlichkeit und Gesundheit gleichgesetzt wird. In Anbetracht der wachsenden Zahl älterer Menschen ist es deshalb auch die Aufgabe der Dermatologie geworden, sowohl das äußere Erscheinungsbild der Haut als auch die physiologischen Funktionen mit fortschreitendem Lebensalter soweit wie möglich zu erhalten.
Das (ewige) Leben, das du so sehnsüchtig begehrst, wirst du niemals erlangen können. Als die Götter den Menschen schufen, gaben sie ihm nämlich den Odem des Todes ein und behielten das Leben für sich zurück. Gilgamesch, fülle deinen Bauch, ergehe dich Tag und Nacht in Freuden. Deine Tage mögen voll von glücklichen Ereignissen sein. Deine Tage und deine Nächte sollen allein aus Gesang und Tanz bestehen. Zieh reine Kleider an, wasche dein Haupt und bade deinen Leib. Betrachte das Kind, das deine Hand ergreift. Umarme dein Weib und genieße die Freuden der Liebe. Nur dies und nicht mehr können die Menschen erlangen.

Aus dem Gilgamesch-Epos
Seit jeher hat das Alter als letzter Lebensabschnitt die Menschheit beunruhigt und gleichzeitig ihre Wissbegierde angeregt. Der Gesundheit und dem langen Leben wurde bereits in alten Kulturen ein hoher Stellenwert eingeräumt. Philosophen, Naturwissenschaftler und Ärzte haben sich lange darum bemüht, Zusammenhänge aufzudecken, den Alterserscheinungen vorzubeugen. In allen Zivilisationen finden sich medizinhistorische Zeugnisse für zwei Hauptrichtungen der Altenheilkunde: Die erste verfolgt prophylaktische Ziele zur Erhaltung von Jugendfrische und Vermeidung der Altersschwäche von Körper und Geist, die zweite hat Heilabsichten und fasst sogar die Verjüngung des alten Menschen ins Auge. Die Beharrlichkeit, mit welcher man über die ganze Zeit mitunter illusorischen Vorstellungen nachging und einem „Jungbrunnen“ nachjagte, 
erscheint bisweilen absurd, beweist aber, dass sich der Mensch nicht ohne Weiteres von bestimmten Fantasien zu trennen vermag. Jeder Chronist ist aber dazu angehalten, sich herabsetzender Urteile über die Vergangenheit zu enthalten. Das Vorkommen von Gold, Menschenblut und Mumienhaut in einem Verjüngungsmittel darf nicht zum spöttischen Lächeln verleiten. Für unsere Vorfahren erfüllte das einen Sinn und entsprach einer Notwendigkeit, deren Ursache zu ergründen ist. Unseren Vorfahren „Irrtümer“ vorzuwerfen wäre ein Anachronismus, denn damit messen wir sie mit den Maßstäben unserer Zeit. Es wäre verfehlt, wenn wir uns durch unsere modernen Kategorien des Denkens den Blick auf das Andersartige in der Vergangenheit verstellen und der Geschichte nicht gerecht würden sowie ihre Eigentümlichkeiten nicht mehr wahrnehmen könnten.

Auch wenn man sich seit der Antike aktiv mit dem Altern auseinandergesetzt hatte, gründete die medizinische Wissenschaft erst Mitte des 20. Jh. die Gerontologie, die sich mit dem methodischen Studium der biologischen Ursachen und Bedingungen des Alterns beschäftigt. Die Gerontologie hat sich zu einer multidisziplinären Spezialität entwickelt, der sich weltweit zahlreiche Forscher widmen, denen es gelungen ist, wichtige Entdeckungen zu machen: die Rolle des oxidativen Stoffwechsels und der freien Radikale, die Mitochondrialtheorie, die Rolle der Hormone und des neuroendokrinen Systems, die Entdeckung von Langlebigkeitsgenen, die Telomertheorie usw.

Die Geriatrie oder Altersmedizin beschäftigt sich indessen mit der Therapie alterstypischer Erkrankungen und der Rehabilitation alter Menschen. Rehabilitation bedeutet in der Geriatrie, den Betagten zu befähigen, seinen Lebensweg zu sehen, zu gehen und zu gestalten.

Im Hinblick auf die demografische Entwicklung, der zufolge sich der Bevölkerungsanteil der über 65-Jährigen bis zum Jahr 2050 verdoppeln wird, kommt heute aber der Prävention eine immer größere Bedeutung zu. Es zeichnet sich also die Tendenz ab, möglichst frühzeitig in die Prozesse einzugreifen, welche zur Verminderung der Funktion und Reservekapazität von Organen und des Gesamtorganismus führen, deren Folge Krankheit und Tod sind.

Man unterscheidet die Primärprävention, die einzusetzen hat, bevor der Alterungsprozess beginnt, die Sekundärprävention, wenn der Alterungsprozess bereits eingesetzt hat, und die Tertiärmaßnahmen bei fortgeschrittener Alterung. Auch wenn auf dem Gebiet der Prävention noch viel Forschungsarbeit zu leisten ist, ist es ein Verdienst der modernen Geriatrie, dass sie aus der Perspektive der Evidence Based Medicine inzwischen in einigen Bereichen gute Fundamente für rationale präventive Strategien liefert, z. B. in der Behandlung des Bluthochdrucks und von Fettstoffwechselstörungen zur Prävention von Herz-KreislaufKrankheiten und Osteoporose-Prophylaxe oder -Therapie zur Prävention von Knochenbrüchen an Schenkelhals und Wirbelkörper.

Während bis vor Kurzem eine fast vollständige Trennung zwischen Gerontologie und Geriatrie bestanden hat, tendieren sie gegenwärtig dazu, einander effektiv zu ergänzen. Aus ihrer Verbindung hat sich die Anti-Aging-Medizin als eigenständige Disziplin entwickelt, die sich die Anwendung wissenschaftlicher Erkenntnisse und medizinischer Technologien im Dienste der Prävention, Früherkennung, Umkehr und Behandlung altersabhängiger Veränderungen und Funktionseinbußen des Organismus zur Aufgabe gemacht hat. Umgangssprachlich sagt man, die Haut sei ein Spiegel der Gesundheit, weshalb ihre Behandlung einen wichtigen Aspekt der Anti-Aging-Medizin darstellt. Ge- sundheit wird oft mit Schönheit gleichgesetzt. In Anbetracht der wachsenden Zahl älterer Menschen ist es Aufgabe der AntiAging-Medizin und Dermatologie, sowohl das äußere Erscheinungsbild der Haut als auch die physiologischen Funktionen mit fortschreitendem Lebensalter soweit wie möglich zu erhalten.

Trotz aller Fortschritte der gerontologischen Forschung und modernen Anti-Aging-Medizin ist nicht zu verkennen, dass während der über 4000-jährigen Auseinandersetzung mit Altern und Tod die wichtigsten Elemente des Anti-Aging dennoch nicht Errungenschaften unserer Zeit sind, speziell im Bereich von Hygiene, Diätetik, Schönheitspflege, Kosmetik, Eigenverantwortung für die Gesundheit, ärztliche Vorsorgeuntersuchung und Psychohygiene.

\section{Gesundheitsprophylaxe, Pflege und Kosmetik im alten Ägypten \\ $\nabla$}

Ägyptens hoher Kultur und besonderen klimatischen Bedingungen sind die ältesten schriftlichen Zeugnisse in Form der Papyri zu verdanken. Im Papyrus Smith erweist sich ein Text mit dem vielversprechenden Titel „Einen Alten in einen Jungen zu verwandeln" als ölhaltige Salbe auf Grundlage der Samen des Bockshornklees, welche unschöne Altersmerkmale der Haut beseitigen soll. Wir wissen, dass der Klee Phytoöstrogene enthält, hormon-aktive Substanzen, die heute in Anti-Aging-Hautcremes angeboten werden.

Als wesentliche Ursache von Krankheit wurden Ukhedu angesehen, von der Stuhlmasse im Darm ausgehende Giftstoffe, die sich im Organismus ausbreiten und die verschiedenen Partien des Körpers erreichen, um dort Krankheit auszulösen. Im Papyrus Beatty findet sich entsprechend eine Sammlung von Rezepten für das Leibesinnere, vornehmlich solche zur Regelung der Verdauung.

Die Papyri mit medizinischen Texten zeigen aber auch, dass viele Darlegungen stark von magischen Vorstellungen durchtränkt waren. Der Gebrauch tierischer Produkte ohne pharmakologische Wirkung war sehr häufig, denn man dachte, dass die Eigenschaften der Lebewesen im Blut ihren Sitz hätten, in den Organen sowie im ganzen Gewebe. Um Haare vor Ergrauen zu schützen, hatte man zum Beispiel die Wahl zwischen verschiedenen Produkten, die von schwarzen Tieren stammten: dem Blut eines schwarzen Kalbs, dem Wirbelknochen eines Raben, dem Blut aus dem Horn eines schwarzen Ochsens oder dem Fett einer schwarzen Schlange. Der Papyrus Leyden hebt die Wirkungskraft von Gold als Verjüngungsmittel hervor. Man war der Ansicht, Gold erhalte die Lebenswärme, weil es in der Astrologie mit der Sonne als dem Ursprung aller Energie in Verbindung gebracht wurde. Dieses Metall, zusammen mit Menschenblut, der Haut von Mumien und dem Fleisch von Giftschlangen waren bis zum 12. Jahrhundert die häufigsten verschriebenen Ingredienzen zum Zwecke der Verjüngung. Die Eigenschaft der Schlangen, ihre Haut zu wechseln und diese damit zu regenerieren, ist wohl der Grund dafür, dass Schlangen sich in der Herstellung solcher Mixturen einer besonderen Beliebtheit erfreuten.

Die Abwehrhaltung des Menschen gegenüber seiner biologischen Begrenztheit ist in der altägyptischen Hochkultur in der Form eines Totenkultes mit ausgefeilter Balsamierungstechnik besonders deutlich sichtbar. Im Darüber-hinaus der Religion weigerte sich der Mensch, seine biologische Begrenztheit anzuerkennen. Die Religion gab nicht zuletzt deshalb maßgebliche 


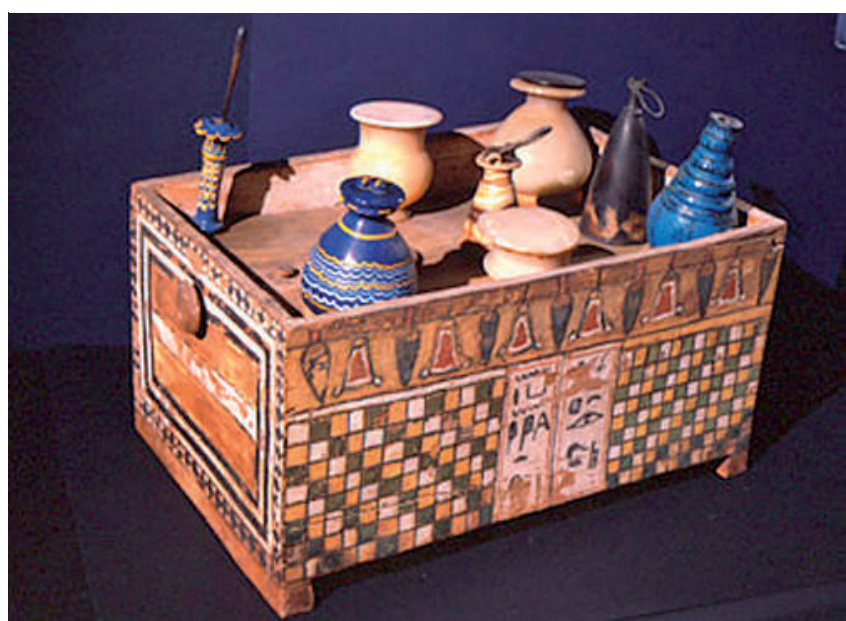

Abb. 1 Altägyptisches Kosmetikkästchen (British Museum).

Impulse für die kosmetische Gestaltung, und Ägypten könnte man geradezu als die Wiege der Kosmetik bezeichnen ( $\bullet$ Abb. 1): Frauen und Männer schminkten Lippen und Wangen in Rottönen, zogen die Brauen mit schwarzem Stibium, gewonnen aus Antimonerz, nach und färbten die Augenlider mit pulverisiertem Malachit grün. Zur Färbung der Haare waren Hennarot und Indigoblau weit verbreitet. Kahlköpfige Männer und Frauen trugen Perücken.

\section{Hygiene, Diätetik und Körperkultur in der griechischen Antike}

Die wissenschaftliche Medizin des Abendlandes wurzelt im alten Griechenland. In einer vollkommen von magischer Mentalität geprägten archaischen Zeit glaubte man, dass die Naturerscheinungen einer bestimmten Ordnung folgten, die beeinflusst werden könnten, sofern man über die entsprechende Machtmittel verfüge. An der Spitze der Völkerstämme standen deshalb Führer mit außergewöhnlicher Macht, denen man den Rang von Gesandten der Gottheit einräumte. Diese bildeten Familien, in denen das metaphysische Wissen weitergegeben wurde. Es entstanden die geheimen Kenntnisse der Mysterien, aus denen eine bestimmte gesellschaftliche Klasse ihre Vorteile zog. Diese „Göttersöhne“ waren zugleich Gesetzgeber, Seher, Zauberer, Dichter und Arzt. Die Medizin selbst stellte zunächst eine Art primitiver, von magischen Vorstellungen durchsetzter Psychotherapie dar, zu der später eine auf den Eigenschaften bestimmter Pflanzen und Mineralien beruhende Heilbehandlung hinzu trat. Der Arzt-Magier griff in das Walten der Natur ein, kannte die geheimnisvollen Eigenschaften von Pflanzen und Mineralien, die dem Kranken die Gesundheit wiedergeben. Zauberei und medizinische Therapie durchdrangen sich gegenseitig, Beschwörungsrituale und ärztliche Behandlung standen eng nebeneinander.

Die Heilung des Tyrannen Iphiklos durch den Zauberer und Arzt Melampus wirft ein Licht auf die Behandlungsweisen der damaligen Zeit: Iphiklos litt unter Impotenz, wünschte sich jedoch inbrünstig, aus eigener Kraft seine Nachkommenschaft zu sichern. Melampus heilte ihn, indem er ihm von einem alten Schwert abgekratzten Rost zu schlucken gab. Er wurde reichlich dafür belohnt. Heilungen, die auf magische Praktiken beruhten, Gunstbezeigungen der Könige mit Erlangung von Reichtum machen die Geschichte jener realen oder legendären Persönlichkeiten aus, die als erste Ärzte im heroischen Zeitalter der Griechen gewirkt haben sollen.

Auf die da und dort isoliert wirkenden Magier folgten die Priester des Asklepioskultes, die ihre Heilkunst in Tempelanlagen ausübten. Der 1260 v. Chr. in Thessalien geborene Arzt Asklepios, der gemäß Pindar „durch das Wort, durch die Elemente und durch das Messer heilte" wurde vergöttlicht, und seine Priester nannten sich Asklepiaden. Ihre medizinischen Kenntnisse waren esoterischer Natur und wurden auf dem Weg der Einweihung weitergegeben. Gefordert war für den Erfolg die bedingungslose Bereitschaft des Kranken, die Regeln des Kultes zu befolgen. Asklepios selbst vertrat den Standpunkt, dass Gesundheit nur dann herbeizuführen sei, wenn man einen Weg zur Beherrschung der Leidenschaften fände.

In der zweiten Hälfte des 8. Jahrhunderts v. Chr. erscheinen die beiden umfangreichsten griechischen Epen, die zugleich erstrangige medizinische Dokument darstellen: die Ilias und die Odyssee des Homer. Homer berichtet in seinen Epen nicht nur in poetischer Weise über den Krieg gegen Troja, sondern entwickelt gleichzeitig eine Deutung der Situation des Menschen in der Welt. Für ihn ist die Welt durch eine Fülle von Gegensatzpaaren bestimmt, wie Alter - Jugend, Götter - Menschen, Worte - Taten usw. Der Krieg als vorherrschendes Thema der Ilias stellt dabei nur eine, wenn auch sehr dramatische Form der Auseinandersetzung dar, in dem sich ein weiterer Gegensatz von eminenter Bedeutung manifestiert, der von Leben und Tod. Homers Götterwelt stellt in der Geschichte der Mythologie ebenfalls eine Neuerung dar: Während die Götter der mythischen Vorzeit erdgebundene, weibliche Mächte waren, die mit den Elementen, die sie darstellten, fest verbunden waren, wie Erde, Zeugung, Blut und Tod, handelt es sich bei den homerischen Göttern des Olymp um helle, personale und betont männliche Gestalten. Der Unterschied zu den Menschen besteht in der Unsterblichkeit der Götter: Sie sind immer jung und strahlend, und der Tod ist ihnen verhasst. Während die Art, in der die Menschen den vorhomerischen Göttern oder Mächten entgegentraten, die Magie war, ist bei Homer davon nicht mehr die Rede. Wir finden keine Spuren magischer Behandlungsweisen mehr in der Homerischen Medizin. Bei Homer ist der Arzt ein Praktiker geworden, der auf Erfahrungen und Tatsachen gründet. Homer zeigt uns das Leben der Ärzte und ihre täglichen Aufgaben auf dem Schlachtfeld und im Lager. In der Ilias erscheint Asklepios nicht mehr als Gott, sondern als ein thessalischer König und Arzt, der es zur Vollkommenheit gebracht hat. Seine Söhne, Machaon und Podaleirios, sind ebenfalls Ärzte, die als Krieger am Kampfgeschehen teilnehmen und gleichzeitig die Verwundeten und Kranken versorgen, der eine als Chirurg, der andere als Allgemeinmediziner.

Nach Homer ist Hesiod der zweite bedeutende Epiker der archaischen Zeit. Er lebte im 7. Jahrhundert v. Chr. In seinem Epos „Werke und Tage“ entwirft Hesiod ein Bild der bäuerlichen Welt, in dem sich Anweisungen zur Gesundheitserhaltung finden. Für ein gesundes Verhalten während drückender Sommertage empfiehlt er:

„Halte dich im Schatten eines Felsvorsprungs auf! ... Drehe das Gesicht in die Richtung, aus der der erfrischende Zephir weht. Schöpfe Wasser aus einer sprudelnden Quelle und nimm auf drei Viertel Wasser ein Viertel Wein ..."

Die Trennung der altgriechischen Heilkunst von den magischreligiösen Praktiken erfolgte im Zeitalter der Naturphilosophen. Einige unter den Naturphilosophen waren selbst Naturforscher 
und Ärzte, die physikalische Gesetze erforschten und diese auf lebende Organismen anwandten. Damit entwickelte sich aus einer naturwissenschaftlich ausgerichteten Philosophie eine wissenschaftliche Medizin, die auf die nachfolgende hippokratische Medizin großen Einfluss haben sollte. Für die Denkart der damaligen Zeit kennzeichnend sind Xenophanes' (um 570 bis um 470 v. Chr.) Überlegungen zur Erkenntnissituation des Menschen:

„Klares hat freilich kein Mensch gesehen, und es wird auch keinen geben, der es gesehen hat / hinsichtlich der Götter und aller Dinge, die ich erkläre. / Denn sogar wenn es einem in außerordentlichem Maße gelungen wäre, Vollkommenes zu sagen, / würde er sich dessen trotzdem nicht bewusst sein: bei allen Dingen gibt es nur Annahme.“

Dennoch endet die Einsicht in die Beschränktheit menschlicher Erkenntnis nicht in der Resignation. Vielmehr wird bei Xenophanes der Gedanke des Erkenntnisfortschrittes aktuell. Die Unzuverlässigkeit der menschlichen Erkenntnis wird zum Ansporn, selbst immer genauer zu forschen und das jeweils für wahr Befundene als vorerst Verlässliches festzuhalten:

„Die Götter haben den Menschen durchaus nicht gleich am Anfang alles enthüllt, / sondern im Lauf der Zeit suchen und finden sie Besseres hinzu.“

Von allen medizinischen Schulen der griechischen Antike ist die Schule von Kos wohl die Berühmteste. Ihren Ruhm verdankt die Schule von Kos vor allem Hippokrates von Kos (460 - 377 v. Chr.). Für die griechische Medizin und die nachfolgende Medizingeschichte des Abendlandes zeichnete sich mit Hippokrates eine entscheidende Wende ab. Hippokrates läuterte die Medizin endgültig von den magisch-religiösen Elementen und orientierte sich an der Beobachtung sämtlicher Aspekte des äußeren Erscheinungsbilds des Menschen. Er lehnte die Auffassung ab, es gebe Krankheiten, die göttlichen Ursprungs seien. Hippokrates fasste den Mensch als Organismus auf, der in Wechselbeziehung zu seiner natürlichen Umwelt steht: Klima, Wasser- und Bodenbeschaffenheit sowie Ernährung und Lebensweise spielen für die Gesundheitserhaltung eine Rolle. Hippokrates entwarf unter Berücksichtigung all dieser Faktoren eine individuelle Diätetik, welche Nahrung, Gymnastik und Sport sowie ein naturgemäßes Verhalten beinhaltete. Im Traktat „Von der Lebensweise“ wird auf die Bedeutung eines angemessenen Verhältnisses zwischen Ernährung und körperlicher Ertüchtigung hingewiesen, außerdem auf die unterschiedlichen Eigenschaften der verschiedenen Getreidesorten und Fleischarten. Krankheit und Gesundheit werden auf das Verhältnis der gegensätzlichen Kräfte Nahrungsaufnahme und körperlicher Beanspruchung zurückgeführt. Wenn sich beide im Gleichgewicht halten, so herrscht Gesundheit, wenn nicht, dann Krankheit:

„Dem Menschen, der Nahrung zu sich nimmt, kann es nicht gut gehen, wenn er nicht gleichzeitig auch seinen Körper durch sportliche Ertüchtigung beansprucht. Ernährung und Sport haben gegensätzliche Qualitäten ... Man muss nicht allein ... diese Qualitäten ... kennen, sondern auch das richtige Verhältnis zwischen dem Maß an körperlicher Ertüchtigung und der Nahrungsmenge, dem Wesen des Individuums, dem Alter ...“

Was das Alter anbetrifft, vertrat Hippokrates die Anschauung, dass die Lebenskräfte des Menschen mit 42 nachzulassen beginnen und das Alter mit 63 Jahren einsetzt. Er riet zur Mäßigung in allen Dingen und Menschen im Alter, ihren gewöhnlichen Tätigkeiten so lange nachzugehen wie sie wollen und ihre Arbeitsgewohnheiten auf keinen Fall jählings zu unterbrechen. Nicht zuletzt überliefert er uns in seinem 2. Buch der Abhandlungen

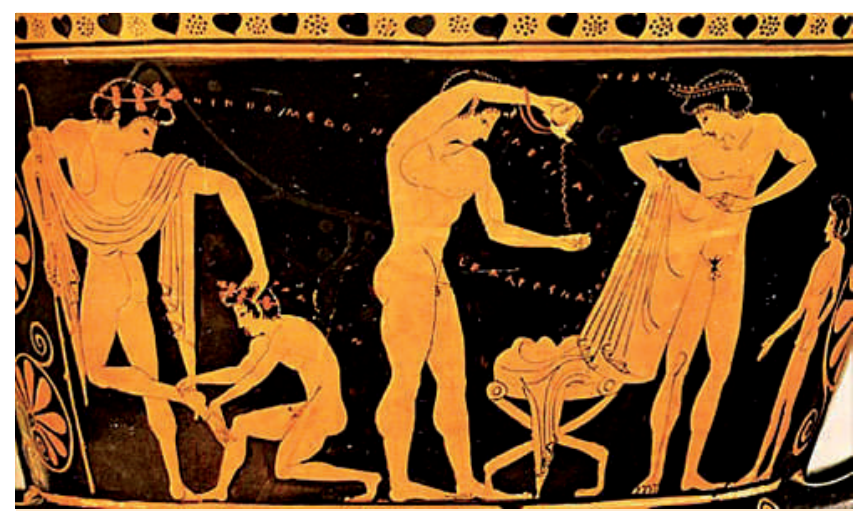

Abb. 2 Körperkultur in der griechischen Antike. Gefäß, Attika, ca. 510 v. Chr.

über Frauenkrankheiten auch kosmetische Rezepturen zur Schönerhaltung der Haut:

„Zur Glättung von Runzeln verreibe man Molybdän in einen steinernen Mörser, gieße abgestandenes Wasser darüber, forme Kügelchen daraus, trockne sie und lasse sie vor dem Gebrauch in Olivenöl zergehen."

Nach verschiedenen Angaben soll Hippokrates von Kos im Alter von 109 oder 112 Jahren gestorben sein. Bald nach seinem Tode begann die Legendenbildung um seine Person.

Im 6. und zu Beginn des 5. Jahrhunderts vor Christus gewann die Körperkultur einen zentralen Platz im griechischen Leben. In den Gymnasien wurden die Schüler neben dem Studium aller Wissensbereiche auch in der körperlichen Ertüchtigung unterwiesen. Die gymnastischen Übungen hatten den Zweck der Erhaltung einer gesunden Konstitution, während die Medizin im Zustand der Krankheit Abhilfe zu schaffen hatte. Um die Schönheit der Körperformen zu erhalten, hatte man strenge Lebensregeln zu folgen, sich einer Diät zu unterwerfen und eine hoch entwickelte Körperpflege zu betreiben. Die Bedeutung der körperlichen Ertüchtigung, wie sie von den Griechen begriffen wurde, lag darin, dem Menschen ein Bewusstsein seines körperlichen Gleichgewichts zu vermitteln ( $\bullet$ Abb. 2). Dann wird ihm von selbst ein Leben der Ausschweifungen, Faulheit und Völlerei zuwider.

In der Nachfolge des Hippokrates wurde die hippokratische Schule gegründet, die in ihrem Selbstverständnis den Lehren des Hippokrates verbunden blieb. Wesentliches Merkmal dieser Schule ist aber, dass sie sich auf die Naturphilosophie von Platon (428 - 347 v. Chr.) stützte. Auch bei Platon sind Krankheiten auf ein Missverhältnis zwischen körperlicher Leistung und Ernährung zurückzuführen, weshalb in seiner Therapeutik wiederum Diät und Gymnastik einen hohen Stellenwert einnahmen. Für Platon war keine Symmetrie oder Disharmonie folgenschwerer als diejenige zwischen Körper und Seele. Heilkunde war für Platon Wissen um das Gesunde. Wichtig erschien ihm, dass der Patient die Therapie mit trägt und damit für den Heilerfolg mitverantwortlich ist. Platon relativierte die Aufgabe und den Einfluss des Arztes zunehmend zugunsten der Eigenverantwortung jedes Einzelnen für seine Gesundheit.

Wie bereits sein Lehrer Platon, sah Aristoteles (384- 322 v. Chr.) die Aufgabe des Arztes darin, den Patienten in Hinblick auf die Eigenverantwortung für die Gesundheit zu beeinflussen. Ziel der Gesundheitserziehung ist dabei Tüchtigkeit und nicht Rohheit: Ein Muskelprotz ohne geistige Reife bleibt eine lächerliche Erscheinung. In Bezug auf das Altern vertrat Aristoteles den Ge- 
danken, dass Altern auf die Aufzehrung einer „inneren Wärme“ zurückzuführen sei, die jedem Lebewesen zu Beginn seiner Existenz innewohnt. Lange vor dem Zeitalter der Bioenergie postulierte er also, dass mit zunehmendem Alter eine fortschreitende Verringerung des Stoffwechsels einherginge.

Die Abfolge hervorragender griechischer Philosophenärzte wurde durch die Invasion der Mazedonier unter Philipp II. unterbrochen. Philipps Sohn, Alexander der Große, gründete 331 v. Chr. mit der Stadt Alexandria einen Handelshafen, der Abendland und Morgenland verbinden sollte. Erst nach seinem Tod 323 v. Chr. übernahm die Dynastie der Ptolemäer in Ägypten die Macht und erhob Alexandria zu einer Kulturmetropole der antiken Welt. Ihr ist eine erneute Blüte der Künste und Wissenschaften im Zeitalter des Hellenismus zu verdanken. Zu dieser Zeit löste die Medizinschule von Alexandria die traditionellen Ausbildungszentren Griechenlands ab. Die Vielzahl der Schüler und ihre Herkunft aus den verschiedensten Landstrichen lassen erkennen, wie sehr das Studium der Medizin damals über den Mittelmeerraum verbreitet war. Als Begründer der medizinischen Schule von Alexandria gilt Herophilos von Chalkedon (330 - 260 v. Chr.), der sich darum bemühte, die Lücken im Werk des Hippokrates durch Forschungsarbeiten auf dem Gebiet der Anatomie, der Physiologie und der experimentellen Pathologie zu schließen. Für Herophilos, der auch Leibarzt Ptolemaios' I. war, schloss Gesundheitsvorsorge gymnastische Übungen und eine Restriktion der Speisen ein.

Schließlich galt auch das letzte gekrönte Haupt in der Dynastie der Ptolemäer, die ägyptische Königin Kleopatra (69 - 30 v. Chr.) in der antiken Welt als kenntnisreiche Ärztin. Sie schrieb ein Buch mit dem Titel Kosmetikon, eine Sammlung von Formeln für die Zusammensetzung von Schminke, Kosmetika und anderen Mitteln zwecks der Verschönerung. Das Werk ist verloren gegangen, aber spätere Autoren beziehen sich auf einige Rezepte daraus, unter anderem Rezepte zum Ondulieren und Färben der Haare.

Durch Misswirtschaft und Thronstreitigkeiten geriet Ägypten in die Abhängigkeit Roms, bis es nach der Schlacht bei Aktium 30 v. Chr. schließlich zur römischen Provinz wurde, womit der Schlussstrich unter die Stellung Alexandrias als Zentrum der hellenistischen Kultur gezogen wurde.

\section{Schönheitspflege, ärztliche Vorsorgeuntersuchung und „Modeärzte“ im alten Rom \\ $\nabla$}

Im alten Rom des 5. bis 3. Jh. v. Chr., bevor die griechischen Ärzte eintrafen, war die Gesundheit eine Angelegenheit der Götter, die bei Krankheiten von den abergläubischen Römern angerufen wurden. Eine medizinische Heilkunde wurde praktisch nicht ausgeübt, und während der Zeit der Republik wurde die Medizin als verächtliche und für einen römischen Bürger unwürdige Tätigkeit angesehen. Der Arztberuf wurde fast ausschließlich von Fremden praktiziert, die man vormittags auf dem Forum zwischen Händlern, Geschäftemachern und Zauberern antraf. Viele waren Scharlatane, die nebenbei noch einen Krämerladen betrieben und geheimnisvolle Pillen, Salben, Pflaster, Räuchermittel, „Gegengifte“ und Pessare verkauften.

Ab dem 3. Jh. v. Chr. begannen griechische Ärzte, sich in Rom niederzulassen. Man nimmt an, dass Archagathos von Peloponnes als erster griechischer Arzt im Jahre 291 v. Chr. nach Rom gekommen war, um dort seine Kunst auszuüben. Trotz der Proteste Cato des Älteren (234-149 v. Chr.), der den Geist konservativen römischen Republikanertums verkörperte, und kein gutes Wort für die griechischen Ärzte übrig hatte, wurde Archagathos das römische Bürgerrecht verliehen und ihm aus öffentlichen Mitteln an einer Straßenkreuzung Behandlungsräume eingerichtet. Cato selbst propagierte eine bäuerliche Naturmedizin mit einer auf einer natürlichen Lebensweise bauenden Diätetik. Da ihm Ärzte als wenig vertrauenswürdig erschienen, hielt er sie sich vom Leibe.

Mit Asklepiades von Prusa (124 - 56 v. Chr.), der sich 91 v. Chr. in Rom niederließ, brach eine neue Ära der römischen Medizin an. Es bedurfte erst eines Mannes von hohem persönlichem Ansehen, umfassendem Wissen und neuartigen Vorstellungen, um den Römern die Würde des bislang missachteten Berufes des Arztes plausibel zu machen. Als gebildeter Mann, der sich zuvor mit Rhetorik befasst hatte, erwarb er sich eine ausgewählte Kundschaft und ließ sich seine Dienste sehr teuer bezahlen. Schnell wurde Asklepiades Freund und Leibarzt bedeutender Persönlichkeiten wie Marcus Antonius und Cicero. Er zeichnete sich im Verschreiben von Behandlungen aus, die dem Geschmack seiner Kunden entgegenkamen. Unter der Devise „toto, celeriter, jucunde“ („sicher, schnell, angenehm“) empfahl er nur leichte Behandlungen entsprechend den Bedürfnissen der Römer, die gerne viel aßen und tranken und sich auch sonstigen Vergnügungen gerne hingaben. Er empfahl Bäder, Massagen und „Bewegung“, die allerdings darin bestand, den Kranken in der Sänfte zu befördern und ihn dabei zu schütteln, ferner „Fastenkuren“, die mit großzügiger Anwendung guten Weines verbunden waren. Alles in allem wusste Asklepiades zu gefallen, und war sehr geschäftstüchtig.

Die Furcht, im Alter die Gesundheit zu verlieren, beschäftigte die Römer zusehends. Römische Denker bemühten sich um eine gesunde Lebensführung: Seneca (4 v. Chr.-65 n. Chr.), der Philosoph der Mäßigung, Selbstbescheidung und des Ausgleichs, sah in Luxus und Unmoral die größten Gefahren für die Gesundheit: „Es ist kein Wunder, dass die Arzneikunde früher weniger wichtig war, als die Körper noch fest und gediegen und die Speisen leicht und noch nicht durch üppige Kochkunst verdorben waren. Seitdem diese aber in Mode ist und tausend Leckerbissen erfunden wurden, um die Gier zu erregen, ist das, was einst Hungernden Nahrung war, den Gesättigten zur Last geworden.“

Wenn Hippokrates einmal festgestellt hatte, Frauen fielen keine Haare aus, und sie kennten keine Fußgicht, so galt dies nicht mehr für die römische Gegenwart:

„Nun verlieren sie ihre Haare und sind an den Füßen krank. Nicht die Natur der Frauen hat sich aber verändert, sondern ihre Lebensweise."

Seneca lehnte städtische Hast ab und sah in der Unrast einen großen Feind der Gesundheit. Viele Menschen benutzten „Abwechslungen als Heilmittel“, in Wirklichkeit fliehe jeder aber nur vor sich selbst!

Aulus Cornelius Celsus (30 v. Chr. - 50 n. Chr.) ist ein Werk zu verdanken, vermutlich zur Regierungszeit des Kaisers Tiberius entstanden, das detaillierte Auskunft über die Praxis der Medizin zur Zeit des römischen Kaiserreiches gibt. Die Schrift De medicina ist eine Sammlung aller medizinischen Kenntnisse seiner Zeit und das erste in lateinischer Sprache verfasste medizinische Werk. Der Hygiene wird ein hoher Stellenwert eingeräumt. Insbesondere die Diät erfährt eine beachtenswerte Würdigung. Celsus teilt die Nahrungsmittel entsprechend ihrem Nährwert in unterschiedliche Kategorien ein, und das römische Gerstenbrot rivalisiert bei ihm mit dem griechischen Weizenmehl. Bei Celsus wird erstmals auch klar, dass die Medizin für die Reichen nicht diesel- 
be ist wie für die Armen: Für dieselbe Krankheit erhielt ein Reicher Aderlässe, Schröpfungen, Senfumschläge oder Einreibungen mit Öl und Schwefel, heiße Getränke und eine angemessene Diät, die gemäß Celsus „unsere Bauern ohne all diese Heilmittel auskurieren, indem sie einfach einen Tee aus Germander trinken“.

Der zweite große lateinische Enzyklopädist Plinius der Ältere (24-79 n. Chr.) trug in seinem Kaiser Titus gewidmeten Sammelwerk Naturalis Historia („Naturgeschichte“) das chemische, botanische und pharmazeutische Wissen seiner Zeit zusammen und setzte sich mit dem Phänomen des Alterns auseinander. Er empfahl, nach dem Essen nicht zu ruhen, da dies zu ungesunder Korpulenz führe. Auch überlieferte er kosmetische Rezepte. Die griechischen Ärzte, die nach Rom gekommen waren und dort reich wurden - Millionäre, so Plinius -, kritisierte er indessen für ihre Mittelmäßigkeit und Habsucht!

Archigenes von Apameia (1. Jh. n. Chr.) war - wie vormals Asklepiades - ein gewandter Praktiker und ein Mann von elegantem Auftreten, dem eine große Klientel aus der Oberschicht schmeichelte. Er lebte in Rom zur Regierungszeit von Trajan. Er zeichnete sich besonders als Frauenarzt aus, der Vertrauter der Damen der Oberschicht gewesen sein und sogar in ihren Intrigen mitgewirkt haben soll. Als Spezialist für Frauenleiden verfasste er ein Traktat über das Färben der Haare und weitere Schriften mit kosmetischen Rezepten.

Archigenes' Nachfolger Heliodorus (2. Jh. n. Chr.) war als Chirurg zwar auf das Kastrieren von Sklaven spezialisiert, Fragmente seiner Schriften weisen aber darauf hin, dass er außerdem Krampfadern operiert, Nasen gerichtet und Lippen verkleinert hat.

Im Laufe ihrer Geschichte wurde in Rom alles erhältlich, was der Schönheit und Gepflegtheit diente: Duftstoffe aus dem Orient, Schminken, Lippenstifte, Schönheitspflästerchen und Perücken. Petronius Arbiter (?-66 n. Chr.), der „Schiedsrichter des Geschmacks“ am Hofe Neros, schreibt im Satyricon, einem Sittenbild Roms zur Zeit Neros:

„Nicht ist Schönheit genug, noch darf, die hübsch will erscheinen, sich begnügen damit, so wie das Volk es wohl tut.

Selbst die Schönere wird besiegt, besitzt eine andre

Witz und Koketterie, liebliches Lächeln und Wort.

Denn die Schönheit beruht durchaus auf künstlicher Hilfe:

fehlt's an Gepflegtheit, so wird nackende Schönheit vergehn."

In den Annales des Tacitus (56-125 n. Chr.) weist eine Passage darauf hin, dass manche Römer sich auch regelmäßigen ärztlichen Kontrolluntersuchungen im Sinne des modernen „Checkup“ unterzogen, während andere über diejenigen spotteten, „welche, nachdem sie die Dreißig überschritten hatten, fremden Rat darüber nötig hatten, was ihrem Körper nütze oder schade“.

Wer nämlich im alten Rom dieses Alter erreicht hatte, durfte mit einer weiteren mittleren Lebenserwartung von mehr als 20 Jahren rechnen.

Neben Hippokrates ist zweifellos Galenus von Pergamon (129-199 n. Chr.) der bedeutendste Mediziner des Altertums. Es war sein erklärtes Ziel, die Lehre Hippokrates, den allein er aufrichtig bewunderte, fortzusetzen. Nachdem er sich zunächst dem Studium der Philosophie gewidmet hatte, wandte sich Galen der Medizin zu, die er mitunter in Alexandria studierte. In Pergamon übte er ab 158 das Amt eines Gladiatorenarztes, bevor er 163 während der Herrschaft des Marcus Aurelius in Rom eintraf. In Rom hatte Galen nicht zuletzt aufgrund seiner dominanten Persönlichkeit sofort Erfolg. Seinen Freundeskreis wählte er sich aus der Elite der römischen Gesellschaft und wurde nacheinander Leibarzt der Kaiser Marcus Aurelius, Commodus, Septimius Severus und Caracalla.
Galen griff die bereits von den Naturphilosophen geäußerte Vorstellung einer mit zunehmendem Alter fortschreitenden Verringerung der Lebensenergie wieder auf, vermutete aber eine Wechselbeziehung zwischen der Verringerung der inneren Wärme und einem Wasserentzug im Organismus des Greises. Das Altern des Menschen wurde mit dem Ölvorrat in der Lampe verglichen, der ständig abnimmt und die Flamme schließlich erlöschen lässt. Diese Reduzierung wurde später auf das Blut bezogen, welches man bald als Träger der inneren Wärme ansah. Im alten Rom sollen sich deshalb Alte beiderlei Geschlechts in die Arena gestürzt haben, um zwecks Verjüngung von dem Blut der sterbenden Gladiatoren zu trinken!

Wie bereits vor ihm Hippokrates, empfahl Galen alten Menschen tätig zu bleiben, damit die Flamme des Lebens nicht erlösche, und maß der Pflege im Alter eine besondere Bedeutung zu: heiße Bäder, Massagen und Körpersalbungen mit Öl und Essig. Wie Celsus, hatte er genaue Vorstellungen von der Qualität der Lebensmittel, aller Fleischsorten und jeder Pflanze, bereitete seinen eigenen Theriak aus 12 bis 64 Bestandteilen und legte besonderes Gewicht auf Diät. Er riet zu leichtverdaulicher Nahrung mit hohem Nährwert: Fisch, mageres Fleisch, frische Feigen und Pflaumen vor den Hauptmahlzeiten zur Stuhlregulierung, Honig, Sellerie und leichter Wein zur Erhöhung der Harnausscheidung. Galen gilt auch als Begründer der Galenik, der Kunst der Zubereitungen auf dem pharmazeutischen und kosmetischen Gebiet. Berühmt ist sein unguentum refrigerans, die aus Bienenwachs, Olivenöl und Rosenwasser bestand. Sie fand bei den römischen Frauen außerordentliches Gefallen und diente ihnen nicht nur als Mittel gegen trockene Haut, sondern auch, um die Spuren des Alterns zu mildern.

Die Medizin in der Zeit vom Tode Galens (210) bis zur Eroberung von Konstantinopel im Jahr 1453 durch die Türken ist geprägt durch den Einfluss der Gräko-Byzantiner. Die bedeutenden Ärzte dieser Epoche waren Gelehrte, die zugleich in das Studium der Philosophie und der Naturwissenschaften vertieft waren, „Philosophen-Ärzte“. Bis zur Einnahme von Alexandria durch die Araber im Jahre 640 fand ihre Ausbildung an der Schule von Alexandria statt. Die Ärzte bereisten zahlreiche Länder, und ihr Wissen wurde durch die Beziehungen zu den verschiedenen Völkern des östlichen Kaiserreiches und häufige Benutzung der Bibliotheken weiter vertieft. Nachdem die Schule von Alexandria während der Herrschaft des Kalifen Omar mit der Zerstörung der Bibliothek den Todesstoß erhielt, musste sich die griechische Medizin nach Konstantinopel zurückziehen und wurde vollständig byzantinisch. Viele Werke der medizinischen Autoren dieser Zeit wurden auf Verlangen der byzantinischen Kaiser selbst verfasst. Antyllos (3. Jh.) war berühmter Chirurg, Arzt und Hygieniker, der unter anderem Operationen von Krampfadern oder des Katarakt (grauen Stars) erfolgreich ausführte. Er bediente sich oft der physikalischen Therapie, speziell der Wasserheilkunde (Hydrotherapie) und gymnastischer Übungen, und empfiehlt in einer Abhandlung unter dem Titel „Was man für seine Gesundheit selbst tun soll“ die Durchführung von Atemübungen.

Jakob Psychrestos (5. Jh.) erhielt vom Kaiser Leon dem Thracier (Regierungszeit: 457-474) den Titel eines „Führers der Ersten Mediziner“. Er erzielte dermaßen erstaunliche Heilerfolge, dass er von seinen Zeitgenossen als „Retter“ geehrt wurde. Reichen Leuten, die infolge von Sorgen, Exzessen und allzu gutem Essen unter Anspannung, nervöser Müdigkeit und Störungen der Verdauung zu leiden hatten, empfahl er eine leichte und erfrischende Diät. 
Alexander von Tralles (525-605), Bruder des Anthemios, Architekten der Kirche der heiligen Sophia von Konstantinopel, trug in seinen „Zwölf Büchern über die Medizin“ nicht nur die gesamten Kenntnisse über die Krankheiten zusammen, die bis zum damaligen Zeitpunkt gesammelt worden waren, sondern verarbeitete auch die eigene, ,in einer langen Praxis bei der Behandlung von Krankheiten erworbene Erfahrung“. Er spricht zwar mit Hochachtung von Hippokrates und Galen, folgte ihnen aber nicht mehr kritiklos, sondern bestand darauf, im Namen der feststellbaren Wahrheit das zur Geltung zu bringen, was sich vom bisherigen Kenntnisstand als abweichend herausstellt:

„Ein Arzt, der für sich behält, was ihm im Innersten als wahr erscheint, begeht eine große Ungerechtigkeit, eine wahrhaftige Gottlosigkeit, er stürzt sich selbst rettungslos in den schlimmsten aller Irrtümer.“

Heilmittel gegen das Alter billigte Alexander von Tralles nicht und schrieb, dass es gegen Alter kein Heilmittel gibt.

Michael Psellos (1018 - 1078) wurde im Laufe seiner Karriere Professor der Philosophie, Staatssekretär, Großer Kammerherr, Erster Minister und Geheimrat des Kaisers. Der geistvolle Vielschreiber, der zweifelsohne über ein enzyklopädisches Wissen verfügte, behandelte in umfangreichen Werken Themen aus der Philosophie, Geschichte und Medizin. Seine Schriften sind bunt und lebendig, aber Psellos selbst blieb ein Theoretiker, der die Wissenschaft vom Schreibtisch aus betrieb. Er schrieb unter anderem eine „Abhandlung über die Wirkung von Nahrungsmitteln“, einen medizinischen „Aufsatz über Bäder“, ein didaktisches „Gedicht in jambischen Versen“, das 1372 Verse enthält, die hygienische und diätetische Vorschriften einschließen, und eine „Abhandlung über die Kräfte von Edelsteinen“, in der er die medizinischen Eigenschaften von Edelsteinen behandelt.

Simeon Seth (11. Jh.) war Leibarzt des Kaisers Michael VII. Dukas. Nachdem er wichtige Titel erlangt hatte, zog er sich in ein Kloster zurück, bevor er 1042 von der Kaiserin Theodora zurück an den Hof gerufen wurde. Sein wichtigstes Werk trägt den Titel „Alphabetische Sammlung über den Wert der Nahrungsmittel“, das er mit folgenden Worten Michael Dukas gewidmet hatte:

„Sobald Ihr die Einwirkungen und Eigenschaften der Nahrungsmittel kennt, könnt Ihr sie für Eure Gesundheit vorteilhafter nutzen. Denn es gibt nichts Kostbareres und nichts Nützlicheres für einen guten Fürsten als Gesundheit und ein langes Leben.“

Demetrios Pepagomenos (13.Jh.) war Arzt von Kaiser Michael VIII. Paleologos (Regierungszeit: 1259-1282). Im Auftrag des Kaisers verfasste er eine „Abhandlung über die Gicht“, das den hohen byzantinischen Würdenträgern, unter denen die Gicht stark verbreitet war, von großem Nutzen sein musste. Demetrios Pepagomenos beschreibt die Gicht als Folge von „überflüssigen Säften " (wir wissen heute, dass es sich hierbei um die Harnsäure handelt), die nicht nur die Hände und andere Gelenke befallen, wo sie Schmerzen und Schwellungen verursachen, sondern auch innere Organe: das seien dann sehr ernste und schwer zu heilende Komplikationen. Die Ursache dieser Krankheit erkannte er in der Lebensart, obwohl er wusste, dass die Krankheit auch erblich sein kann. Er hielt fest: „Ein Mensch, der sich gesund halten will, isst und trinkt wenig."

Unter vielen talentierten Autoren hat die griechisch-byzantinische Medizin außergewöhnliche Persönlichkeiten hervorgebracht, denen nicht nur Fortschritte in der Lehre der Krankheitserscheinungen (Semiologie), der Diagnostik und der Therapie von Krankheiten zu verdanken sind, sondern auch der normalen, für die Erhaltung der Gesundheit erforderlichen Hygiene: Sie nutzten die therapeutischen Möglichkeiten und verordneten
Diäten, Nahrungshygiene, Hydrotherapie, Thermal- und Mineralquellen, Klimawechsel und Spezialkuren, alles was die heutigen „Wellness“-Institute anbieten.

\section{Gesundheitsbildung und Fürstenregimen in der arabischen Welt \\ $\nabla$}

Die medizingeschichtliche Epoche, die innerhalb der islamischen Welt mit der Hedschra, der Auswanderung des Propheten Mohammed nach Medina im Jahre 622 und bis zum Beginn der Neuzeit reicht, wird als das Zeitalter der „arabischen Medizin“ bezeichnet. Der Überlieferung nach soll Mohammed (570-632) selbst als Arzt gewirkt haben und versorgte während der Eroberungszüge die Verwundeten in eigens für sie errichteten Zelten. Im Zuge der von seinen Nachfolgern fortgesetzten Eroberungen fielen weite Gebiete des Oströmischen Reichs in die Hände der Araber. Hier stießen die arabischen Beduinen auf die intellektuell und wissenschaftlich hoch stehende Kultur der Byzantiner, von denen sie sehr schnell lernten. In diese Zeit fällt zwar der Niedergang großer, den gesamten Wissensschatz der damaligen Zeit vermittelnden Schulen, wie die berühmte Schule von Alexandria (640), und dennoch: Die Gesamtheit der geistigen Errungenschaften des östlichen Mittelmeerraumes in der Spätantike blieb nur durch die Vermittlung der Araber erhalten, ohne deren Bemühungen dieses Kulturerbe verloren gegangen wäre. Indem die großen Werke ins Arabische übersetzt wurden, stieg die arabische Welt zur Hauptträgerin des damaligen Wissens auf, inklusive der auf Gesundheitserhaltung und Körperpflege bezogenen Gebiete der Physiologie, Hygiene, Ernährung, Gymnastik und Massage. Unter dem Einfluss der arabischen Kultur wurden Türkische Bäder mit Massage zu einer gewohnten Einrichtung auch in der abendländischen Welt bis zum Ende des Mittelalters. Als zu diesem Zeitpunkt die Städte immer dichter bevölkert wurden und das Wasser sich verknappte, nahm ihre Zahl sukzessive ab.

Im Westen indessen gewann bei der Verschmelzung der Kulturen und Weltanschauungen inzwischen das Christentum die Oberhand, und die in der Zeit der Kirchenväter betonte Leibfeindlichkeit wirkte sich auf die Körperpflege und Kosmetik negativ aus. Der Kirchenvater Tertullianus (160 - 230) erklärte jede Art der persönlichen Verkleidung als eine Sünde vor Gott. Er argumentierte, dass Schminke, Puder und Perücken eine Form solcher Verkleidung seien und, da nicht angeboren und damit von Gott gegeben, Erfindungen des Teufels. Kaiser Theodosius I. (Regierungszeit 379-395) verbot öffentliche Bäder und alle nackt ausgeführten sportlichen Aktivitäten. Die geistige Führerschicht verdammte alle kosmetischen Mittel. Kaiser Justinian I. (527-565), der sich als Erneuerer des Römischen Reiches verstand und auch im religiösen Bereich aktiv war, versetzte den „Heiden“ einen Schlag, indem er 529 die von Platon eingerichtete Akademie in Athen schließen ließ.

Der Islam zeigte sich demgegenüber weltoffen und zerstörte nicht das, was er vorfand. Das 9. Jahrhundert war durch eine intensive Übersetzertätigkeit gekennzeichnet, die sich im 10. Jahrhundert fortsetzte. Die Ärzte wurden von den Herrschenden hoch geachtet, beherrschten mehrere Sprachen und widmeten sich nicht ausschließlich der Heilkunst, sondern setzten sich auch mit den meisten anderen damals bekannten Naturwissenschaften auseinander.

Avicenna (980-1037) griff die großen Themen der Philosophie des Aristoteles auf und versuchte eine allgemeine und überge- 
ordnete Wissenssynthese aller Wissenschaften untereinander herzustellen. Die Vermischung der verschiedenen wissenschaftlichen Gattungen führte Avicenna dazu, die vier Elemente des Universums, die vier Körpersäfte, die vier Jahreszeiten, die vier Charaktere jedes Körpers, die vier Zustände des Menschen usw. schematisch in einen Zusammenhang zu bringen. Die Medizin erscheint innerhalb dieses genialen Versuchs als großartiges Geistesspiel, das allerdings für die praktische Medizin völlig unergiebig war. Die Einführung von Astrologie und verworrener abstrakter Begriffe in die Medizin verdeutlicht, wie weit sich Avicenna mit seinen hochkünstlichen, geistigen Konstruktionen von der praktischen Medizin entfernte. Und dennoch, Analogieschlüsse und symbolische Deutungen dieser Art, in die auch die Sterne einbezogen wurden, sollten im Laufe des gesamten Mittelalters immer häufiger werden. Wie unfruchtbar dies für den Fortschritt der Medizin war, ist indessen allgemein bekannt. Das Quanun fit'tibb („Gesetze der Heilkunst“), auch unter dem Namen Canon medicinae bekannt, stellt Avicennas medizinisches Hauptwerk dar, das bald zu universeller Bedeutung gelangte und bis ins 17. Jahrhundert fester Bestandteil des Lehrplans medizinischer Fakultäten der abendländischen Universitäten war.

In der Altenpflege empfahl Avicenna heiße Bäder, Massagen mit Öl, gemäßigte Leibesübungen, Diät aus Ziegen- oder Eselsmilchund Pflanzenprodukten, abgelagerten Rotwein, Abführmittel und ein ruhiges und ausgeglichenes Leben.

Averroës (1126 - 1198) bemühte sich wie Avicenna um das Werk des Aristoteles, allerdings weil ihm der Ruhm, der dem Aristoteles-Kommentator Avicenna zukam, ungerechtfertigt erschien. Mit Ausnahme der Offenbarungslehre, unterwarf er alles der freien Kritik und betonte, dass der Philosoph und der Naturwissenschaftler in geistiger Freiheit nach der Wahrheit suchen müssten. Sein Denken stellte somit einen wichtigen Schritt in Richtung auf eine unabhängige, experimentelle Naturwissenschaft dar.

Das Schrifttum des jüdischen Arztes Maimonides (1135-1204) kreist um das Spannungsfeld zwischen Schöpfungsoffenbarung und Naturerkenntnis. Wie die Christen zu dieser Zeit bewegten sich die arabischen Ärzte in einem Spannungsfeld zwischen dem Verdacht auf Gottlosigkeit auf der einen Seite und hoher Wertschätzung ihrer Wissenschaft auf der anderen. Man kann verstehen, dass sie alles unternahmen, um die Religion auf ihrer Seite zu haben, und Fragen zur berufsspezifischen Ethik spielten in der arabischen Medizin nicht zuletzt deshalb eine große Rolle. In seinem Menschenbild trachtet Maimonides nach einem Ausgleich zwischen dem materiellen Körper und dem Geist und kommt zum Schluss, dass beide für die Gesundheit von gleicher Bedeutung seien. Maimonides beschäftigte sich mit Fragen von weiser Lebensführung und vernünftiger Daseinsstilisierung. Sein Hauptwerk More Nevuchim („Leitung der Ratlosen“) stellt eine Synopsis von Wissenschaft und Religion dar. Ein philosophisches Verständnis der Offenbarung hielt er nicht für möglich, wenn man nicht auch die Natur des Menschen erschließt, und diese ist nur durch die Medizin möglich. Maimonides sah deshalb für jeden gebildeten Menschen die Beschäftigung mit der Medizin als selbstverständlich an.

Als Krankheitsursachen sah Maimonides erbbedingte oder umweltbezogene, soziale Faktoren oder Schädigungen aufgrund eigener Verhaltensweisen an. Als Aufgabenbereiche der Heilkunst bezeichnete er die Anleitung der Gesunden zu vernünftiger Lebensführung, die sachgemäße Behandlung der Kranken, sowie die Beratung derer, die nicht ganz gesund, aber auch nicht richtig krank sind. Da der Mensch zur Beherrschung der Natur angelegt ist, hat er die Pflicht, entsprechend richtig zu handeln: Diätetik und Hygiene als medizinische Grundprinzipien werden zum Modell einer Stilisierung des Alltags. Die Grundzüge der Krankheitslehre des Maimonides sind somit unmittelbar verbunden mit seiner Theorie der Gesundheitserhaltung. Gesundheitsbildung wird zum Ausdruck gebildeter Lebensführung. Gesundsein ist für den gebildeten Menschen das Maß seiner Weisheit. Die sich weise verhalten „lachen dem Tod ins Gesicht“!

Maimonides' Speisediätetik lässt sich in drei Grundsätzen zusammenfassen: „niemals ohne Hunger essen, niemals ohne Durst trinken, die Ausscheidungen niemals aufhalten“. Für den Sultan entwarf Maimonides ein „Fürstenregimen“:

„Schon bei Sonnenaufgang oder kurz davor soll man aufstehen. Man nehme zwei bis drei Unzen von Hydromel-Trank (aus Zucker und Wein) und ruhe eine Weile. Darauf reite man aus, im leichten Ritt und möglichst ohne Unterbrechungen; anschließend betreibe man eine sorgsam gesteigerte Gymnastik, damit die Glieder warm und der Geist frisch werden. Vor dem Frühstück halte man eine kurze Ruhepause ein. Als Nachspeise gibt es Pistazienkerne mit Rosinen oder getrocknete süße Mandeln. Danach folgt wieder ein kurzes Schläfchen, das bei langsamen, gedämpftem Saitenspiel besonders erholsam ist. Der übrige Tag diene der Lektüre, der Konversation, der Bildung des Geistes und der Erheiterung des Gemüts. Vor dem Abendmahl folgt ein Schlückchen Honigwein, danach das gute Abendessen. Daraufhin lade man zur Unterhaltung Sänger ein, lege sich ... zur Ruhe, nachdem man den Sängern die Anweisung gegeben, Spiel und Vortrag allmählich zu dämpfen und nach Erreichen des Tiefschlafs ganz einzustellen.“

\section{Psychohygiene und Makrobiotik im christlichen Mittelalter \\ $\nabla$}

Mit dem Zerfall des islamischen Reiches im 11. Jh. gewann Europa wieder an kultureller Bedeutung. Die argwöhnische sunnitische Orthodoxie der türkischen Vorherrschaft unterdrückte jede intellektuelle Regsamkeit. In Europa wurden indessen in Salerno, Bologna, Padua und Montpellier Medizinschulen gegründet.

Nachdem das Weströmische Kaiserreich mit der Absetzung des Kaisers Romulus Augustulus 476 durch den Heermeister Odoaker dem Ansturm der Barbaren endgültig unterlegen war, und politisches Chaos und Machtkämpfe zwischen religiösen und politischen Doktrinen zum schlechten Nährboden für die Naturwissenschaften wurden, war nurmehr in den Klöstern geistiges Schaffen lebendig, wenn auch mit wenig Originalität. In der mittelalterlichen Medizin wurden die Lehren der griechischen Ärzte übernommen, die von Übersetzungen der arabischen Medizin überliefert wurden, vor allem die Philosophie Aristoteles' durch die Vermittlung Avicennas. Die Medizin stellte keine eigenständige Wissenschaft dar, sondern war eng an eine Kultur gebunden, in der es nicht darum ging, die Wahrheit zu finden, sondern den Glauben als unumstößliche Wahrheit vernunftmäßig zu begründen und verstehbar zu machen. Der Mensch und alles Kreatürliche ist nur durch Gott und um Gottes Willen da. Als Kreatur des göttlichen Willens hat der Mensch die Aufgabe, dem Willen des Schöpfers zu entsprechen, den er in seinem göttlichen Wort offenbart hat. Zur obersten Tugend wird damit Demut im Verhältnis zu Gott, und zum Verwerflichsten die Vermessenheit, Gott gleich sein zu wollen. Das bedingte eine veränderte Wertskala der von den alten Griechen gepriesenen Tugenden, die teil- 
weise geradezu als Hoffart oder „glänzende Laster“ erschienen. Damit wurde das Studium der Natur, die man als ein für das Heil des Menschen wenig wichtiges Abbild des allein erstrebten Unsichtbaren betrachtete, zweitrangig. Es genügte in diesem System das Symbol zur allumfassenden Erklärung. Da die Philosophie dieser Zeit aus der Unterweisung und Erziehung der Geistlichkeit in den Klosterschulen erwachsen ist, wird sie als Scholastik bezeichnet.

Petrus Hispanus (1210-1277) gilt als einer der bedeutendsten Scholastiker, der gleichzeitig Arzt und Philosoph war, und der einzige Mediziner, der je zum Papst gewählt wurde (Johannes XXI.). Für ihn diente die weise Kontrolle der Affekte der Gesundheitserhaltung, und nicht deren Beseitigung, denn sie gehören notwendig zum Leben, als Ausdruck der Verzweiflung des Menschen über seine Endlichkeit.

Gerade in der päpstlichen Kurie, der Petrus Hispanus bereits vor seiner Wahl zum Papst nahe stand, hielt die Kunst der „Lebensverlängerung in Gesundheit" oder Makrobiotik einen wichtigen Stellenwert inne. Da allgemein das Lebensalter der Menschen, im Vergleich zu den alttestamentarischen Patriarchen, abzunehmen schien, breitete sich bei den Theologen, die darin eine Strafe Gottes vermuteten, Resignation aus. Von Noah bis Abraham nahm die Lebensdauer auf besorgniserregende Weise ab, so dass letzterer nur noch 175 Jahre alt wurde! Zur Zeit Jesus Sirachs erreichte der Mensch höchstens hundert Jahre, und der Psalmist nennt als Normalalter des Menschen 70 und „wenn er kräftig ist“ 80 Jahre. So verwundert nicht, dass Papst Alexander III. 1177 seinen Leibarzt nach Indien sandte, um das sagenhafte Land des christlichen Priesterkönigs Johannes aufzusuchen, wo eine geheimnisvolle Quelle ewige Jugend versprach und ein wundersamer Stein „nur Christen und solche, die es werden wollen“, heilte!

Die mittelalterliche Gelehrsamkeit war von Legenden durchtränkt, und der ganze Aberglaube über Gestirne, Fabeltiere und wundersame Heilpflanzen wurde in den Bestiarien egalweg für wahr gehalten. Hier sei nur die Legende vom Vogel Caladrius erwähnt, der sich am Hofe der Könige aufhielt und unfehlbar Heilung oder Tod voraussagte und außerdem mit therapeutischen Kräften ausgestattet war. Auch die medizinische Praxis unterlag stark dem Einfluss von Zauberei, Zahlenmagie und Reliquienglaube.

Angesichts dieser Unzulänglichkeit gründeten klerikale und weltliche Ärzte im 11. Jh. zusammen die Schule von Salerno, die sich bald einen glänzenden Ruf erwarb. Constantinus , der Afrikaner“ (1015 - 1087) war ein geduldiger Übersetzer, der in seinem 22-bändigen Lehrwerk das Lehrgebäude der Schule von Salerno durch Verschmelzung des Erbes von Hippokrates und Galen mit dem Gedankengut der arabischen Medizin von Grund auf gestaltete. In seinem Werk widmet er sich den Problemen der ärztlichen Ausbildung, den Grundlagen der Anatomie und Physiologie, Fragen der Diät und Hygiene sowie der Wissenschaft von den Heilkräutern.

Johannes von Mailand war zugleich Arzt und Poet, der mit seinem Gedicht Flores medicinae („Die Blumen der Medizin“) die Ideen der Schule von Salerno weit über die Grenzen hinaus in ganz Europa bekannt machte. Das große Echo, das das Gedicht fand, verdankt es der Tatsache, dass es nicht für Eingeweihte geschrieben war. Es besticht durch seine Einfachheit und eine vollkommene Klarheit, und handelt von den Vorzügen der einfachen Dinge. Den Schwerpunkt des Gedichts bilden Probleme der Diät und der Hygiene sowohl für den physischen als auch für den moralischen Bereich. Das Gedicht ist einem König gewidmet:
„Für den König der Engländer schrieb die ganze Salerner Schule Willst du ohne Schaden, willst du gesund leben

Vermeide schwere Sorgen und betrachte Zorn als profan.“

Eine weitere Grundschrift der Diätetik aus der Medizinschule von Salerno stellt das Regimen Sanitatis Salernitatum dar. Zahlreiche Sentenzen sind aus diesem Text in die Alltagssprache der europäischen Nationen eingegangen, wie zum Beispiel: „Nach dem Essen sollst du ruhn oder tausend Schritte tun. "Wie sehr Physiologie auch Psychologie bedeutet, wird in einer weiteren Forderung deutlich: „Besser als ein Arzt sei die dreifache Regel: Ruhe, Heiterkeit, Mäßigkeit.“

Auch die Schönheitspflege wurde wieder zu einem Teil der Diätetik: In einer der legendären salernitischen Ärztin Trotula (11. Jh.) zugeschriebenen Schrift De passionibus mulierum fand sich ein Kapitel über die Hautpflege. Der Medizinprofessor Wilhelm von Saliceto (13. Jh.) verschrieb Mittel gegen Fettsucht und Krampfadern. Sein Zeitgenosse Gilbertus Angelicus verfasste eine Kosmetik des Gesichts und der Haare. Michele Savonarola gab Ratschläge zur Verkleinerung oder Vergrößerung der Brüste. Den Einfluss des Regimen Sanitatis der Salerner Schule findet sich wieder in einem Buch mit dem Titel „Die Erhaltung der Gesundheit" von Taddeo Alderotti (1223 - 1295). Dieser lehrte in Bologna, wo im 11. Jh. eine Universität eingerichtet wurde, aus der in der zweiten Hälfte des 12. Jh. die ebenfalls berühmte Medizinschule von Bologna hervorging. Alderotti, Leibarzt des Papstes sowie des Dogen von Venedig, räumte dem Zustand der Psyche für den Gesundheitszustand eine herausragende Bedeutung zu: Kranke sollten eine heitere Stimmung suchen, was durch Erinnerungen an angenehme Erlebnisse oder die Betrachtung schöner Dinge möglich sei.

Bereits zu Beginn des 13. Jh. war Bologna von Studenten aus ganz Europa überbevölkert, und als deren Freiheiten durch die Regierung bis zur Unerträglichkeit beschränkt wurden, verließen viele Bologna und gründeten 1228 die Universität von Padua. Einer der prominentesten Gelehrten der Universität von Padua war Pietro d'Abano (1250-1315). In seinem Werk Conciliator differentiarum philosophorum et praecipue medicorum („Der Versöhner zwischen Philosophen und Ärzten“) macht er eine enge Beziehung zwischen Philosophie und Medizin deutlich. D’Abanos wissenschaftliche Methode erfordert strenge Objektivität und somit vom Beobachter höchste Aufmerksamkeit zur genauesten Erfassung aller Symptome. Glauben und Vernunft versuchte er in Einklang zu bringen und verlangte vom Arzt einen großen Ermessensspielraum, da sein Denken durch kein Dogma gefesselt werden dürfe. In diesem Sinn und Geist versuchten seine Schüler beispielsweise die medizinisch wirksamen Substanzen im Wasser der Heilquellen von Abano zu finden, und es gelang ihnen, Salze zu extrahieren und durch Verdampfen zu kristallisieren. Auf diese Weise wich die Alchimie allmählich auch einer wissenschaftlichen Chemie. Die objektive Methode d'Abanos sollte bahnbrechend für die neue Medizin werden, wie bereits d'Abano in seinem Conciliator prophetisch angekündigt hatte: totus mondus commutatur („die ganze Welt wird verändert“). So kündigte sich gegen Ende des Mittelalters eine neue Ära wissenschaftlichen Beobachtens und Experimentierens an, die im Laufe der Renaissance ihre Fortsetzung erfahren sollte. Als Professor der Medizin und Philosophie erfreute sich d'Abano allgemeiner Hochachtung und wurde zum gefragten „Modearzt“ von Prinzen und Reichen. Dabei vertrat er die Ansicht, dass das Vertrauen des Patienten zum Arzt eine wichtige Rolle für die Wirksamkeit der Behandlung und den Heilerfolg spielt. 


\section{Psychohygiene, Zeitökonomie und Gerontocomia in der Renaissance \\ $\nabla$}

In der Renaissance erfuhren Kunst und Wissenschaft einen Wandel. Mit der Entwicklung eines neuen Verständnisses der Wirklichkeit rückte der Mensch in den Mittelpunkt, und die Argumentation richtete sich nicht mehr nach den autoritativen Texten. Die ersten Humanisten bekämpften leidenschaftlich die wissenschaftlich-scholastische Orientierung der mittelalterlichen Medizin.

Rein „geistig“ sollte die Sehnsucht nach Gesundheit befriedigt werden: Petrarca (1304-1374) vertrat in seinen Hauptschriften die „seelische Gesundheit“, die er mit geistiger Reife, Menschlichkeit und sittlicher Vollkommenheit gleichsetzte, und kritisierte die „Gottlosigkeit“ der Mediziner, deren Gesundheitsbemühung gekennzeichnet war von Handwerk und „gespreizter Sprache“. Nicht manierierte Rezepte, sondern das „einfache Leben“, die vita solitaria, wie sie von Mönchen und Einsiedlern gepflegte wurde, sowie der Rückzug in die Gesellschaft bedeutender Autoren wie Cicero oder Cato stellten nach ihm einen Erfolg versprechenden Weg zur Gesundheit dar. Die Gesundheit sah Petrarca vor allem durch die urbane Rastlosigkeit gefährdet. Nur das einsame Leben des solitarius brachte den vollkommenen Einklang mit sich selbst.

Auch der Universalgelehrte Leon Battista Alberti (1404-1472) propagierte den sinnvollen Wechsel zwischen öffentlicher Betätigung und Rückzug ins Private. Zeichen der gesunden Lebensführung war für ihn eine perfekte Zeitökonomie, die der Getriebenheit des Städters entgegensteht: „Ein fleißiger Mensch geht immer langsam!“

Der Philosoph und Arzt Marsilio Ficino (1433-1499) sah indessen in drei Betätigungen Gefahren für den Intellektuellen: zu häufiger Beischlaf, ein Übermaß an Wein und Speisen, sowie Nachtarbeit. Im Übrigen hielt Ficino die alte Vorstellung bei, dass der „Baum des Körpers“ mit fortschreitendem Alter immer mehr austrocknete, und befürwortete, Flüssigkeiten junger Menschen zu sich zu nehmen, um dem Wasserentzug des Gewebes entgegenzuwirken. Er empfahl, das Blut junger Leute mit einer oder zwei Unzen Zucker zu mischen. Die Anwendung von Muttermilch sollte ebenfalls der Verjüngung dienen. Das Beste sei,

„ein junges, fröhliches und schönes Mädchen mit ausgezeichneter Gesundheit zu finden, um ihr bei Vollmond von der Brust zu saugen!“

Tomaso Rangone publizierte 1550 eine Papst Iulius III. gewidmete Schrift „Wie man das Leben des Menschen über 120 Jahre hinaus verlängern kann“. Exemplarisch nimmt er Bezug auf das lange Leben der alttestamentarischen Patriarchen und vieler Kirchenväter. Die Gesundheit galt als Vorbedingung humanistischer Lebensart und konnte gemäß Rangone mittels ruhiger Lebensweise und sorgfältiger Speisenwahl erhalten werden. Auch Empfehlungen - in der Tradition der Viersäftelehre - zur künstlichen Korrektur mittels Erbrechen und Aderlässen, zur Bekämpfung der Pest, zur Heilung der Syphilis, zur Kosmetik und Gymnastik fehlten gemäß dem umfassenden Charakter des Werkes nicht.

Gabriele Zerbi (?-1505) prägte 1489 den Begriff Gerontocomia, womit er die Kunst bezeichnete, den Prozess des Alterns aufzuhalten, und plädierte für einen neuen Beruf, den Gerontocomus, eine Art Gesundheitsberater für alte Menschen, dessen Pflichtenheft er ausführlich beschreibt. Zerbi kann demnach als „Begründer“ einer Fachdisziplin des „Anti-Aging“ angesehen wer- den. Er fügte allerdings den Ratschlägen von Galen und Avicenna keine wesentlichen neuen Erkenntnisse hinzu: die Wichtigkeit, ein gemäßigtes Klima zu wählen, die Notwendigkeit, sich im Winter gut zuzudecken und im Sommer Schwitzen zu vermeiden, mäßige Bewegung nach den Mahlzeiten, Einsamkeit zu vermeiden und die Vorzüge von Hühnerbouillon für geschwächte Greise. Wenn sich sexuelle Beziehungen auch schlecht untersagen lassen, so sollten Aphrodisiaka doch vermieden werden. Auch finden die alten Heilmittel der Magie, wie Destillate von menschlichem Blut, Puder von wertvollen Steinen und Gold noch Erwähnung, Zerbis Verdienst ist es aber, dass er diesen keine Bedeutung mehr zuschrieb.

Henri de Mondeville (1250-1325), der in seinen Schriften erstmals eine klare Trennung zwischen medizinischen Therapien für krankhafte Zustände und (kosmetischen) Behandlungen zwecks Verschönerung eine klare Trennung vollzieht, behandelt in seinem Buch zur Chirurgie im Kapitel über die Verschönerung der Männer und Frauen auf eindrückliche Weise die Entfernung von Haaren, die plastische Brustkorrektur, die Haar- und Gesichtspflege sowie die Verjüngung der alten Frauen.

Dennoch war die Kosmetik nicht im modernen Sinn verwissenschaftlicht. In vielen Hinsichten stand sie der Alchimie mit ihren toxikologischen Risiken noch nahe, und es haftete ihr noch viel Geheimniskrämerisches an.

Als wichtiger Vertreter der deutschen Medizin zu dieser Zeit und Alchimist sei der Schweizer Theophrastus Bombastus von Hohenheim oder Paracelsus (1493 - 1541) genannt, der die Iatrochemie begründete, eine Frühform der Chemie im Dienste der Medizin. Trotz seines für seine Zeit fortschrittlichen Denkens, haftete auch seinem Wirken Mysteriöses an. Sein Denken ist vor dem Hintergrund von Renaissance, Humanismus und Reformation zu sehen. Seine radikale Kritik an der althergebrachten Lehre fand ihren Kulminationspunkt am 20. Juni 1527, als er in symbolischer Handlung Avicennas „Kanon der Medizin“ ins Basler Johannisfeuer warf und dabei ausrief „Ins Feuer mit dir, auf dass alles Schlechte sich mit dir in Rauch auflöse!". Diese Geste galt als Herausforderung an die alte Schule und als Appell an die Vernunft und Erfahrung als einzige Erkenntnisquellen. Nichtsdestotrotz finden sich in Paracelsus' naturphilosophisch-magischer Heilkunde alchimistische und astrologische Überlegungen sowie religiöse und dämonologische Spekulationen, die ihn unter dem Strich nicht als jenen eindeutigen Wegbereiter des wissenschaftlichen Fortschritts ausweisen, wovon die übliche Verehrung seiner Persönlichkeit ausgeht. Der Legende nach soll Paracelsus für Diane de Poitiers, die Mätresse Heinrich II. von Frankreich (1519-1559), gegen Haarausfall eine Flüssigkeit mit Blut einer Wöchnerin, Ingredienzen von einem getöteten Neugeborenen und Schlangengift gemischt haben!

Gemäß dem großen Humanisten Erasmus von Rotterdam (1466-1536) erhält die Quinta essentia („fünfte Essenz“ oder "Quintessenz“) die Gesundheit,

„denn es ist keine Fabel, sondern durch etliche Zeugen verbürgt, dass der Mensch mittels der fünften Essenz die Altersschwäche wie eine Schlangenhaut abstreifen und sich wieder verjüngen kann.“

Die Herstellung der „Quintessenz“ war, neben derjenigen des Goldes, während Jahrhunderten ein Hauptziel der Alchimie. So nimmt es auch nicht wunder, dass seit dieser Zeit lange viele Scharlatane ihr Unwesen trieben. 


\section{Säkularisierung des Körpers und Entmytholo- gisierung der Leiden im Zeitalter der Aufklärung $\nabla$}

Im Zeitalter der Aufklärung legte René Descartes (1596 - 1650) in seinem Discours de la méthode pour bien conduire sa raison et chercher la vérité dans les sciences („Abhandlung über die Methode des richtigen Vernunftsgebrauches“. Erscheinungsjahr: 1637) die Grundlagen zum rationellen wissenschaftlichen Denken. Ein für die Medizin überaus folgenreiches Ereignis war die durch Descartes vorgenommene Teilung des Organismus in einen dem Regelwerk eines Automaten nahe stehenden Leib und die von diesem unabhängige Seele. Dieser „Cartesianische Dualismus“ bedeutete faktisch die Säkularisierung des Körpers. Von nun an standen physikalische Konzepte im Mittelpunkt der Gesundheitstheorien. Mithilfe technischer Mittel wurde die Körperwelt erforscht. Durch die Messung von Pulsfrequenz, Körpertemperatur und anderer physikalischer Größen vertiefte eine neue Generation von Iatrophysikern die Cartesianischen Theorien.

François de la Boe (Sylvius) (1614-1672) definierte die Gesundheit als optimales Verhältnis der Säure- und Alkalibildung im Körper, wobei auch mechanistische Vorstellungen eine Rolle spielten. Vielen schien damit der Weg in eine „seelenlose“ Medizin gewiesen, und eine Entmythologisierung des Körpers und der Leiden wurde begründet.

Die Erfindung des Mikroskops um 1590 erleichterte auch die medizinische Forschung. Wichtiger Vertreter der mikroskopischen Forschung waren Antoni van Leeuwenhoek (1632-1723) und Robert Hooke (1635-1702), zu dessen ersten Studienobjekten Flaschenkorken gehörten. Die mikroskopisch kleinen Hohlräume, die er in den Flaschenkorken beobachtete, bezeichnete er als „Zellen“. Dieser Begriff sollte später zum Grundbegriff der Zytologie, der Wissenschaft vom Bau und von den Funktionen der Zelle, und der Zellularpathologie, einer auf die Zellenlehre basierenden Krankheitslehre, werden. Ihr zufolge ist in der Auffassung des Organismus als „Zellenstaat“ die Ursache aller Krankheiten in einer Störung der einzelnen Zellen zu suchen, im Unterschied zur alten „Humoralpathologie“ von Galen, bei der als Ursache aller Krankheiten eine fehlerhafte Mischung der Körpersäfte postuliert wurde.

Bald versuchte man auch, Beziehungen zwischen den Alterserscheinungen und anatomischen Veränderungen herzustellen: Im 17. Jh. sezierte William Harvey (1578-1657) einen Greisen, den hundertjährigen Thomas Parr, aber erst Giovanni Battista Morgagni (1682-1771) und Gerhard van Swieten (1700-1772) verglichen klinische Beobachtungen und anatomische Befunde systematisch miteinander. Morgagni gibt präzise Beschreibungen alterstypischer Veränderungen wie Katarakt, Prostatahyperplasie, Arteriosklerose und Lungenemphysem wieder, und van Swieten stellt fest, dass es mit zunehmendem Alter zu einer Abflachung der Bandscheiben kommt.

Allerdings beherrschten der Cartesianismus und die Iatrophysiker die Gesundheitslehren des 17. Jh. nicht unangefochten. Selbst der Iatrochemiker Johann Baptist van Helmont (1577 - 1644), der wie viele seinesgleichen die Zeit angekommen sah, das „Buch der Natur“ und damit auch die Geheimnisse der Gesundheit zu dechiffrieren, räumte der Seele als lebenserhaltendes Prinzip eine zentrale Bedeutung ein. Ihre Stabilität sei schon deshalb notwendig, um die Gesundheit nicht durch Imaginationes („Einbildung“) zu gefährden: Bereits die Furcht vor der Pest könne die Erkrankung bewirken! Auch die Geisteshaltung der Ärzte kritisierte Helmont:
„Die Vernunft ist von der Wahrheit radikal verschieden. Sie hat die Ärzte nur dazu gebracht, nicht mehr zu heilen, sondern zu reden.“ Auch der Philosoph Jean-Jacques Rousseau (1712 - 1778) vertraute den Ärzten nicht und schätzte deshalb ihre Hilfe und Arzneien gering ein:

„Wenn die Beobachtung lehrt, dass Arzneien weder festere Gesundheit noch längeres Leben verleihen, dann ist diese Kunst schon schädlich, weil sie nicht nützlich ist und Zeit, Dinge und Menschen umsonst beansprucht."

Ein langes Leben findet sich gemäß Rousseau nur bei Menschen, die viel Bewegung gehabt und Mühe und Arbeit haben ertragen müssen. Seine Devise lautete: „Wie ein Bauer arbeiten und wie ein Philosoph denken." Aber auch die Gesundheit des naturgemäß lebenden Menschen sei gefährdet, doch fürchtet er nicht den Tod wie seine „zivilisierten“ Zeitgenossen, sondern er stirbt in Frieden, weil er nicht durch Ärzte, Philosophen und Priester beeinflusst zu sterben verlernt hat!

Die Zeit war also durch eine zunehmende weltanschauliche Verunsicherung, religiöse Zweifel und Sinnsuche gekennzeichnet. Es war auch eine Blütezeit der Geheimkulte, die die Schaffung einer neuen Welt, Gesellschaft und Gesundheit verkündeten. Schon das Eingeweiht sein galt den Geheimbündlern als Weg zur Gesundung, und die Suche nach dem „Stein des Weisen“ schloss diejenige nach Glück, Erfüllung und körperlicher Gesundheit mit ein. Als Schutzmittel galten geheime Elixiere, die in alchimistischen Apotheken gemischt wurden. Der medizinische Alltag wurde von vielerlei „Heilern“ geprägt: Wahrsager, Gesundbeter, Wunderheiler, Exorzisten, Okkultisten und Zauberer versprachen die Erhaltung der Gesundheit und langes Leben, während Kabbalisten, Paracelsisten und Rosenkreuzer bewusst esoterisch gehaltene Beweisführungen lieferten. In Paris wurde 1681 ein Mittel feilgeboten, das durch Öffnung der Hautporen krankheitserregende Einflüsse entweichen ließ, und ein angeblich 400-jähriger Venezianer mit Namen „Galdo“ galt als lebender Erfolgsbeweis!

Christoph Wilhelm Hufeland (1762 - 1836) verurteilte die mittelalterlichen Techniken der Gesundheitssuche, wie geheime Kräfte, Stein der Weisen, Kabbala, Astrologie, Alchimie, den Paracelsismus und die Wunderheiler des 18. Jh., und nimmt in seiner „Makrobiotik. Die Kunst, das menschliche Leben zu verlängern“ Rousseaus Philosophie, dass die Natur im Unterschied zur „Zivilisation“ großzügig Gesundheit verleihe, wieder auf:

„Wenn man sich denkt, wie wenig ein Naturmensch auf den Südseeinseln von Krankheiten weiß, und dagegen nun ein europäisches pathologisches Kompendium hält, wo sie regimenter- und kompanienweise aufmarschieren, und ihre Zahl sich auf viele Tausende beläuft, so erschrickt man davor, was durch Luxus, Sittenverderbnis, unnatürliche Lebensart und Ausschweifungen möglich geworden ist. Viele, ja wirklich die meisten dieser Krankheiten sind unsere eigene Schuld.“

Hufeland postulierte als Grundursache allen Lebens eine „Lebenskraft", die die seit Descartes vorherrschenden mechanistischen Theorien nicht erklären konnten. Die Lebenskraft stelle die „feinste, durchdringende, unsichtbarste Tätigkeit der Natur“ dar, wie man sie in einem Samenkorn finde, das mehrere Jahre ein „gebundenes Leben“ enthalten kann. Werde sie entzogen, verfalle der Körper den Gesetzen der toten chemischen Natur und gehe in Fäulnis über. Hufeland sah in der Stärkung der Lebenskraft den Schlüssel zum langen Leben. Jeder Mensch verfüge über ein bestimmtes Quantum dieser Kraft, das durch ungesunde Lebensweise vermindert wird. Unmäßigkeit im Essen ebenso wie „raffinierte Kochkunst“, Untätigkeit, üble Laune, 
Furchtsamkeit, maßlose Leidenschaften, auch Neid und Missgunst konsumieren die Lebenskraft und hindern die Restauration. Wiederholt preist Hufeland das Land- und Gartenleben, wo alles auf Erhaltung des Lebens hinwirke. Die Medizin selbst suche zwar durch Stärkung und andere Mittel den Menschen auf den höchsten Grad seiner physischen Fähigkeit zu erheben. Dabei zeige die Makrobiotik, dass es auch hier eine Grenze gibt, und „ein zu weit getriebener Grad von Stärke das Mittel werden kann, das Leben zu beschleunigen und folglich zu verkürzen“. Hufeland sah gleichzeitig ein, dass eine „gute physische Herkunft", was die Lebenserwartung betreffe, ebenfalls von Bedeutung sei, gebe es doch ganze Familien, „in denen das Altwerden ein Familienprivilegium war", und nahm damit den heutigen Forschern nach „Langlebigkeitsgenen“ die Erkenntis einer genetischen Grundlage zum langen Leben vorweg.

Im 18. und 19. Jh. nutzten schließlich viele Romantiker das Kranksein als Befreiung von den bürgerlichen Pflichten zugunsten eines ausschließlich der Kunst gewidmeten Lebens. Die Auffassung der Schwindsucht war die einer Krankheit, die bevorzugt Reiche, Junge, Frauen, sensible und zerbrechliche Geschöpfe befiel und mit Lebensüberdruss zusammenhing. Sie mutete als ätherische Schönheit an und befriedigte manche Eitelkeit bis in das Zeitalter des Dandytums hinein. Im leidenden Zustand sah die Bohème den Beweis einer höheren Sensibilität und Kreativität, überhaupt einer sublimen Existenz. Gesundheit im Sinn einer vitalen Dynamik und das Ideal einer einfachen und robusten Lebensart galt bei den Snobs als obsolet und geschmacklos!

\section{Reduktion des Gesundheitsbegriffes auf messbare Größen in der europäischen Gesundheitslehre des 19. Jahrhunderts}

$\nabla$

Der endgültige Bruch der Heilkunde mit den romantischen Gesundheitskonzepten zur Mitte des 19. Jh. wird mit Rudolf Virchow (1821 - 1902) in Verbindung gebracht, dessen 1845 gehaltene Rede „Über das Bedürfnis und die Richtigkeit einer Medizin vom mechanischen Standpunkt" eine kritische Auseinandersetzung mit dem romantischen Geist bedeutete. Die Menschheit hatte nach ihm das religiöse und philosophische Zeitalter überwunden und war in eine Phase des positiven Wissens übergegangen. Für Virchow offenbarten sich Krankheit und Gesundheit im Zustand der Körperzellen und ihrer Beziehung zueinander, und er gilt als Begründer der Zellularpathologie. Heilung bedeutete Regulation der gestörten Verhältnisse auf der Ebene der kleinsten bekannten Körperbausteine, den Zellen. Dabei verband Virchow den Gesundheitsbegriff eng mit Normwerten.

Die Geschichte der europäischen Gesundheitslehre bis zum Ende des 19. Jh. zeichnete sich durch eine Reduktion des Gesundheitsbegriffes auf messbare Größen aus. Eine systematische anatomisch-klinische Methode fand Mitte des 19. Jh. definitiv auch in die Altersmedizin Eingang: Clovis-René Prus (1793-1850) lieferte 1840 den ersten Versuch einer statistischen Untersuchung der Pathologie des Greises im Altenheim. Maxime Durand-Fardel (1816-1899) untersuchte in seiner Traité clinique et pratique des maladies des viellards („Klinische und praktische Abhandlung über die Krankheiten der Greise“. Erscheinungsjahr: 1854) die Alterskrankheiten, getrennt nach den einzelnen Organen.

Jean-Martin Charcot (1825 - 1893) griff das alte Dilemma wieder auf, ob es sich beim Alter um eine Krankheit an sich handle oder um einen Normalzustand. Er versuchte diesem Konflikt zu ent- kommen, indem er drei Arten von Krankheiten alter Menschen unterschied: als erste die Krankheiten des organischen Zerfalls, wie seniler Kräftezerfall (Kachexie), Osteoporose, zerebrale Atrophie, Arteriosklerose etc., als zweite die Krankheiten, die man in allen Altersstufen antrifft, die jedoch beim alten Menschen eine unterschiedliche Entwicklung und Prognose aufweisen, beispielsweise die Pneumonie, und als dritte schließlich die Krankheiten, denen die alten Menschen sogar besser widerstehen als die jungen, zum Beispiel der Typhus und die Tuberkulose. Bei dieser Gelegenheit weist Charcot in aller Deutlichkeit darauf hin, dass sich mit zunehmenden Alter eine klare Trennung zwischen Physiologie und Pathologie immer schwerer ziehen lässt.

Lorenz Geist (1807-1867) verfolgte im deutschen Sprachraum denselben Weg. Sein Werk „Klinik der Greisenkrankheiten“ stützt sich auf die Autopsie von 500 Personen. Wohl hatten die Anatomen festgestellt, dass bestimmte Organe mit zunehmendem Alter ihr Gewicht verringern, insbesondere die quer gestreifte Muskulatur, die Leber, die Nieren, die Milz und die Geschlechtsdrüsen. Mit dem Schwund dieser Organe vollzieht sich gleichzeitig eine Verminderung ihrer Leistungsfunktionen. Erstmals schlug Geist physiologische Tests vor und unternahm selbst Untersuchungen der Lungenventilation mittels Spirometrie.

In Großbritannien lag der Schwerpunkt der Altersforschung indessen auf dem Herzen. Bereits die altägyptischen und griechischen Mediziner der Schule von Alexandria schrieben dem Herzen die Rolle eines „Schrittmachers“ zu - nach Aristoteles Sitz der „inneren Wärme“ -, auf dessen natürliche Rückbildung das Altern zurückzuführen sei. Man war der Auffassung, das Herz nehme bis zum Alter von 50 Jahren an Gewicht zu, und danach verringere sich sein Gewicht.

Wie bereits die alten Ägypter, vertrat Ilja Metschnikow (1845-1916), 1908 Nobelpreisträger für Medizin, die Auffassung, dass das Altern auf eine langsame chronische Vergiftung durch spezifische „Toxine“ zurückzuführen sei, welche von den Darmbakterien produziert würden, weshalb er das Altern durch Sauermilch und Joghurt zu bekämpfen suchte.

Charles-Edouard Brown-Séquard (1817-1894) war schließlich der Ansicht, dass die Geschlechtsdrüsen verantwortlich für die Bewahrung der körperlichen und geistigen Jugendfrische seien. 1849 hatte der deutsche Physiologe Arnold Adolf Berthold (1803 - 1861) darauf hingewiesen, dass ein Stoff existieren müsse, der von Drüsen produziert wird und an anderen Orten eine Wirkung entfaltet, indem er kastrierten Hähnen Hodengewebe einsetzte und damit das Reifen eines Hahnenkammes bewirkte. Später wurde der Begriff „Hormon“ (von horme = griech.: „Anregung“) geprägt. Brown-Séquard unternahm 1889 mit wässrigen Extrakten aus den Hoden von Hunden und Kaninchen Verjüngungsexperimente an sich selbst vor $(\bullet$ Abb. 3).

In den frühen 1920er-Jahren wurde festgestellt, dass sich durch die Unterbindung des Samenstranges die Keimzellen im Hoden zurückbilden, während sich die Testosteron bildenden Zellen vermehrten. In der Folge wurde diese Operation in der falschen Annahme einer erhöhten Testosteronproduktion propagiert und über Hundert Wiener Universitätsprofessoren, darunter Sigmund Freud (1856 - 1939), unterzogen sich diesem Eingriff!

Eine Reihe experimenteller Pseudo-Forschungen schloss sich diesen Versuchen an, und etliche Kliniker, selbst aus dem ärztlichen Establishment, nutzten die öffentliche Leichtgläubigkeit auf einträgliche Weise aus. Eine lärmende Werbung für Verjüngungstherapien, wie die Transplantation der Hoden von Men- 


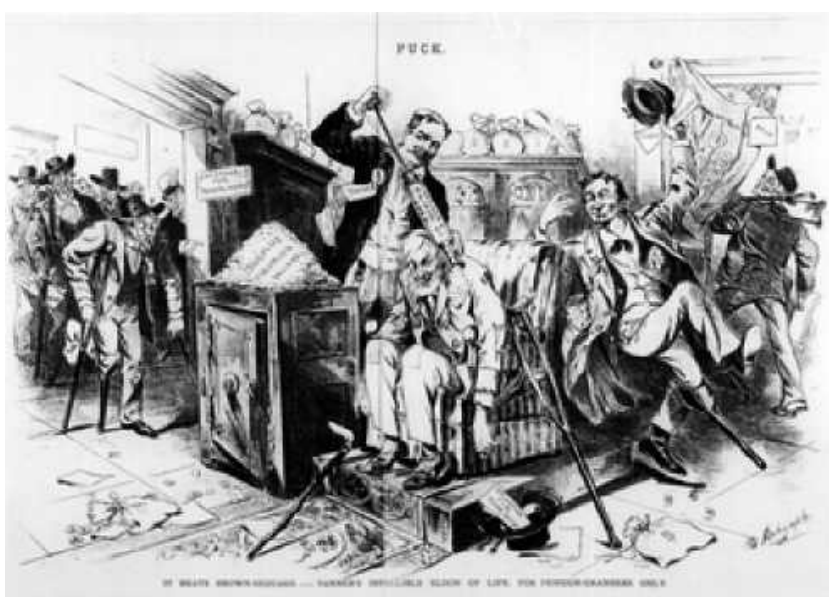

Abb. 3 Geburtsstunde der „Opotherapie“ (Gewebesafttherapie) im 19. Jh.: Karikatur von Louis Dalrymple, Puck, 28. August 1889.

schenaffen, Durchtrennung oder Ligatur des Samenstranges, die Einpflanzung von Plazentateilen und Injektionen von Embryozellen von Rindern, die alle auf zumindest zweifelhaften experimentellen Grundlagen beruhten, trugen dazu bei, am Anfang des 20. Jh. Biologen und Kliniker zunächst von einer seriösen Altersforschung abzuhalten.

Erst 1945 wurden wieder ernst zu nehmende Forschungen aufgenommen, und zwar zunächst über die Modalitäten des endokrinen Alterns. Der russische Endokrinologe Vladimir Korenchevsky (1880-1959), der nach Großbritannien emigriert war und von 1920 bis 1945 am Lister Institut für Präventivmedizin tätig war, gründete 1945 in Oxford die erste Gerontological Research Unit und 1950 die International Association of Gerontology. Ihre seither regelmäßig durchgeführten Kongresse trugen viel zum Fortschritt der neuen Disziplin bei. Gleichzeitig entstanden weltweit zahlreiche fachliche Periodika, die eine rasche Veröffentlichung der ständig wachsenden Zahl von Arbeiten über das Altern ermöglichten: Zeitschrift für Altersforschung (1938), Journal of Gerontology (1946), Geriatrics (1946), Journal of the American Geriatrics Society (1951), Gerontologia (1957), Experimental Gerontology (1964), Age and Ageing (1972), Mechanisms of Ageing and Development (1972), Experimental Ageing Research (1975) usw.

\section{Einzug der Anti-Aging-Medizin in die kommerzielle Welt der Moderne \\ $\nabla$}

1993 wurde die American Academy of Anti-Aging Medicine (Präsident: Ronald Klatz) gegründet, die heute weltweit über 10000 Mitglieder zählt und alljährlich einen internationalen Fachkongress in Las Vegas, zuletzt mit mehr als 4000 Teilnehmern in einem der dortigen Großhotels, ausrichtet.

Unter dem Stichwort Anti-Aging wird relativ gesunden und funktionell uneingeschränkten Menschen ab fünftem bis sechstem Lebensjahrzehnt nach eingehender Analyse ein maßgeschneidertes Programm aus Hormonen, Vitaminen und Diätempfehlungen angeboten, um Lebensqualität bis in ein hohes Alter zu erhalten. Die Anti-Aging-Protokolle basieren auf der Hypothese, dass zwischen einer altersabhängigen Abnahme zirkulierender Hormone und der Funktionseinbuße ein ursächli- cher Zusammenhang bestehe, weshalb Hormontherapien im Vordergrund stehen. Diese werden ergänzt durch passive und aktive Körperanwendungen, Sport, Diätprogramme, Vitamintherapien und nach Bedarf auch durch kosmetisch-chirurgische Eingriffe.

Das in den Vereinigten Staaten von Amerika gegründete Konzept des Anti-Aging verfolgt zweifelsohne primär ein kommerzielles Interesse. In den letzten Jahren haben auch in Europa AntiAging-Kliniken Fuß gefasst, deren Honorar für die Therapie sich in einem Bereich von bis zu 25000 Euro im Jahr bewegt. Potenzielle und kapitalkräftige Kunden werden mit Erfolgsberichten von Anwendern aus dem eigenen Institut umworben. Gewöhnlich bleibt unklar, was nun wirklich gewirkt hat, die Hormontherapie, der veränderte Lebensstil, oder beides. Bisher gibt es keine auf kontrollierte Studien basierenden Daten, die belegen, dass die Anti-Aging-Protokolle die physiologischen, altersabhängigen Veränderungen verhindern und die individuelle Lebenserwartung verlängern würden.

\section{Abstract}

\section{In Quest of the Fountain of Youth \\ $\nabla$}

Everywhere and at all times old age with its afflictions has preoccupied mankind. Philosophers, scientists, and physicians have always endeavored to unravel the process of aging, to effectively avert the signs and symptoms of aging, though with limited success. All civilizations bear testimony for two main streams of undertakings: the first encompasses preventive measures to preserve youthfulness and avert senile infirmity, the second curative procedures and measures to rejuvenate the old. The tenacity with which illusory beliefs have been pursued on the quest of the fountain of youth may seem absurd to us at times. They simply prove that man cannot let go of certain phantasies. Nevertheless, in all great cultures of the past a number of remarkable hypotheses have been set up, that since then have found confirmation. Examples are found in the field of hygiene, nutrition, body culture, beauty care, cosmetics, personal responsibility for one's own health, medical checkup, psychohygiene, and time management, all important elements of current anti-aging. Today, antiaging as a novel medical specialty pursues the prevention, early diagnosis, reversal, and treatment of age-related loss of function and alterations of the organs and the organism. The condition of the skin plays an important role in anti-aging medicine, since good looks are equaled to youthfulness and health. With respect to the growing number of older people, it has become a challenge for dermatologists to preserve both a good outer appearance and intact physiologic functions of the skin as far as possible with growing age.

\section{Literatur}

1 Bergdolt K. Leib und Seele. Eine Kulturgeschichte des gesunden Lebens. München: C. H. Beck, 1999

2 Schott H (Hrsg). Meilensteine der Medizin. Dortmund: Harenberg, 1996

3 Toellner R (Hrsg). Illustrierte Geschichte der Medizin. Salzburg: Andreas \& Andreas, 1986

4 Trüeb RM. Anti-Aging. Von der Antike zur Moderne. Darmstadt: Steinkopff, 2006 